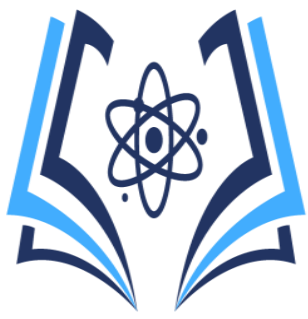

\title{
Prevención y atención de conductas adictivas en Adolescentes del cantón Jipijapa
} \section{of the Jipijapa canton \\ Prevención y atención de conductas adictivas}

Prevention and care of addictive behaviors in Adolescents

\author{
Mg. Doris Susana Delgado Bernal ${ }^{(1)}$ \\ Mg Delia Georgina Bravo Bonoso ${ }^{(2)}$ \\ Mg. Bárbara Miladys Placencia López ${ }^{(3)}$ \\ Lic. Silvia Gabriela Cáceres Palma ${ }^{(4)}$ \\ ${ }^{1}$ Universidad Estatal del Sur de Manabí, doris.delgado@unesum.edu.ec, Orcid: https://orcid.org/0000- \\ 0001-5614-2567
}

${ }^{2}$ Universidad Estatal del Sur de Manabí, delia.bravo@unesum.edu.ec, Orcid: https://orcid.org/0000-00034787-8403

${ }^{3}$ Universidad Estatal del Sur de Manabí, miladys.placencia@unesum.edu.ec, Orcid: https://orcid.org/00000003-4970-2305

${ }^{4}$ gabixi@outlook.es, Orcid: https://orcid.org/0000-0003-2558-5984

Contacto: doris.delgado@unesum.edu.ec

Recibido: 12-08-2021

Aprobado: 10-9-2021

\section{Resumen}

Las drogas son un problema a nivel mundial, muchos adolescentes comienzan a consumirla por curiosidad, influencia del grupo de amigos, depresión u otros problemas dentro de la sociedad, los riesgos que puede ocasionar el consumo de estas sustancias se transforman en problemas familiares, económicos, muchos de los consumidores llegan a cometer actos ilícitos, dentro de la sociedad para poder adquirir estas sustancias El presente trabajo de investigación pretende determinar el comportamiento del consumo problemático de sustancias adictivas en adolescentes de dos áreas urbanas del cantón
Jipijapa. El método de estudio es de tipo descriptivo, analítico, para poder obtener resultados eficaces, entre técnicas utilizadas se tiene la observación, entrevista realizada casa a casa, en donde se pudo evidenciar los diferentes comportamientos que tienen los adolescentes ante la sociedad, además, el consumo de drogas legales, como el alcohol y el tabaco siguen siendo los más utilizados.

Palabras claves: Drogodependencia, comportamiento, adicción, incidencia, autocontrol 


\section{HIGIA DE LA SALUD}

\section{Abstract}

Drugs are a worldwide problem, many adolescents begin to consume it out of curiosity, influence of the group of friends, depression or other problems within society, the risks that the consumption of these substances can cause are transformed into family and economic problems, Many of the consumers come to commit illicit acts within society in order to acquire these substances. This research work aims to determine the behavior of the problematic use of addictive substances in adolescents in two urban areas of the canton of Jipijapa. The study method is descriptive, analytical, in order to obtain effective results, among the techniques used there is observation, an interview conducted from home to home, where it was possible to demonstrate the different behaviors that adolescents have before society, in addition, the consumption of legal drugs, such as alcohol and tobacco continue to be the most used.

Key words: Drug dependence, behavior,

addiction, incidence, self-control

\section{Introducción}

La historia de las adicciones va unida a la historia del hombre. Fumar cigarrillos, beber alcohol, mascar hojas de coca, esnifar preparados psicoactivos, beber pócimas, fumar marihuana, utilizar el opio para el dolor, etc., son ejemplos bien conocidos de algunas de las sustancias que el hombre ha utilizado a lo largo de la historia o sigue utilizando. La adicción es una enfermedad
ISSN 2773-7705

Periodo. Julio - Diciembre 2021

Vol. 5, Nro. 2, Publicado: 2021-12-31

primaria, que afecta al cerebro, constituida por un conjunto de signos y síntomas característicos. El origen de la adicción es multifactorial involucrándose factores biológicos, genéticos, psicológicos, y sociales.

El fenómeno de las drogas a nivel mundial representa una problemática cada vez más compleja, dinámica y multicausal, que genera daños en la salud, en la convivencia social y seguridad ciudadana, pues desintegra a la sociedad y, sobre todo, su núcleo central que es la familia. Esta situación problemática demanda un abordaje integral, multidisciplinario, inmediato y basado en evidencia científica, con el involucramiento activo de la sociedad a través de sus distintos actores. La información de la problemática en nuestra realidad nacional, alerta acerca de aspectos como el consumo de drogas en edades tempranas, el relativo fácil acceso a las mismas y la determinación de las zonas con mayor incidencia del fenómeno socio económico de las drogas.

Las sustancias aditivas son un serio problema de salud pública en el mundo y los países en desarrollo cada vez va en aumento afectando a todas las familias. A partir de los años sesenta, el consumo se transformó en una preocupación mundial, particularmente en los países industrializados sobre todo por la alta cantidad de riesgos que pueden ocasionar a la salud, la economía, a los problemas jurídicos, familiares, teniendo serias implicaciones en la violencia, delincuencia y el terrorismo internacional Guaranda Moreira , 2018) este consumo excesivo causa diversos tipos de problemas, dentro de las familias y en la sociedad, pues las personas que la consumen pueden llegar a ser violentas o hacer cosas ilícitas por conseguir estas sustancias. 
El consumo de sustancias adictivas constituye un fenómeno social que afecta a los adolescentes, ya que es una etapa en el que se comienza a despertar hacia la edad adulta, ellos realizan un consumo experimental y recreativo, especialmente del alcohol, el tabaco y el uso de otras sustancias que con el tiempo los adolescentes se vuelven dependientes para poder sobrellevar su diario vivir. Es por ello que las medidas de prevención son muy importantes para reducir la oferta y demanda de drogas (Tumbaco Quimis, 2018)

La Organización Panamericana de la Salud (OPS, 2015) plantea que cada año ocurre la muerte de 3 millones de personas en el mundo debido al consumo nocivo de alcohol, que representa un 5,3\% de todas las defunciones. Esto indica un incremento sobre el consumo de bebidas alcohólicas en los individuos y la sociedad, lo que trae como consecuencias un aumento en las adiciones por esta sustancia química, teniendo en cuenta la incidencia en el organismo humano y sus afectaciones pueden estar dirigidas principalmente a los órganos más importantes empezando por el sistema nervioso central, lo que conlleva a que el sujeto presente graves problemas en su salud. Por lo tanto, el prevenir el consumo de alcohol desde edades tempranas propicia la reducción de riesgos para la vida en adolescentes y jóvenes.

Por otra parte, en el ámbito familiar se nombran algunos factores como: el modo de enfrentar los problemas a nivel familiar, y dentro de estos, la falta de apoyo religioso y de la búsqueda de apoyo profesional, conjuntamente con las dificultades de comunicación intrafamiliar (Cid Monckton \& Pedrão , 2011); las pautas de interacción familiar, como la comunicación deficiente, los frecuentes conflictos, la baja
ISSN 2773-7705

Periodo. Julio - Diciembre 2021

Vol. 5, Nro. 2, Publicado: 2021-12-31

cohesión, el excesivo castigo o el consumo de los propios padres (Jimenez, Murgui, Estevez, \& Musitu, 2007). La familia emerge como núcleo desencadenante de conductas de riesgo, si el adolescente la percibe como un ambiente hostil busca un grupo que le brinde seguridad, pero al carecer de una referencia fiable de sus propios atributos, presenta un proceso de desadaptación provocándole el rechazo de otros. Esto deriva en la exposición a factores de riesgo como el consumo de drogas, la promiscuidad y conductas violentas ( Gómez Cobos, 2018).

En Colombia, de acuerdo a lo concluido en el informe final del estudio nacional de consumo de sustancias psicoactivas, del Observatorio de Drogas de Colombia, (ODC), es importante el consumo de alcohol en un $20 \%$ por una población entre 12 y 17 años, pese a estar prohibida la venta a esta población, y dado el riesgo que genera el consumo de esta sustancia en dichas edades correspondientes a la pubertad y la adolescencia, lo que representa un mayor esfuerzo tanto en la conciencia social, como en las políticas que regulen la venta de dichas sustancias a menores de edad. En este mismo informe se encuentran incrementos globales en el uso del cigarrillo, marihuana, bazuco, éxtasis, heroína y cocaína entre la población descrita, siendo este aumento en comparación al año 2008, paso del $8.8 \%$ al $12.2 \%$ en 2013 , lo que representa una preocupante cifra de tres puntos porcentuales (Observatorio de Drogas de Colombia, 2018)

En el Ecuador existe una tendencia creciente de drogodependencia a cocaína, marihuana y heroína que afecta a adolescentes de 12 a 17 años de edad debido al fácil acceso de estas sustancias en todos los ámbitos en los que se desenvuelve el ser humano ocasionando problemas mentales, de 
comportamiento, de conducta, y mal funcionamiento del sistema nervioso central (Ponce Bacusoy, Suarez Martinez, \& Pincay Parrales, 2021)

La adolescencia es sin lugar a dudas una de las etapas más críticas de la vida del ser humano, puesto que es durante esta etapa, que se llegan a experimentar diferentes cambios tanto en aspectos físicos, emocionales, sociales, intelectuales. Son precisamente estos cambios, los que dan origen a complejas situaciones en la vida cotidiana de los adolescentes, para quienes resulta verdaderamente complicado, el proceso de asimilación de estos y a la vez los procesos relacionados con el desarrollo de su propia personalidad, así como el modo de relacionarse con sus padres.

Es sabido que la salud es un tema de mayor importancia en procesos que se construyen a diario con interacción de todos, por lo cual, no debe ser ajeno a nuestro estudio, la explicación de la posición del psicoanálisis frente a temas tan relevantes como las adicciones en los jóvenes adolescentes de nuestro cantón, de igual manera entender que estos jóvenes adolescentes, presentan situaciones tales como aburrimiento, atonía vital, fracaso escolar, uso y consumo de sustancias psicoactivas, conductas agresivas, dudas y miedos sobre conductas sexuales, dificultades de comunicación y el difícil control de emociones tales como el miedo, la ira, ansiedad, tristeza, celos, y otros, que difícilmente pueden ser resueltos por profesionales de la salud, en los primeros tamizajes realizados sobre los jóvenes, por lo cual se hace necesario de la intervención de profesionales en psicología y más específicamente terapeutas en psicoanálisis especializados en el manejo de adicciones.
Periodo. Julio - Diciembre 2021

Vol. 5, Nro. 2, Publicado: 2021-12-31

Esta investigación, se hace con el propósito de hacer un estudio sobre el aporte del psicoanálisis frente a las adicciones en los adolescentes, servirá como fuente de consulta a padres, profesionales de la educación, psicólogos, terapeutas, y lectores en general que coincidan con esta búsqueda de respuestas ante esta problemática, además permite implementar programas, campañas y proyectos orientados a la intervención en la misma institución de estudio, así como también en otras instituciones formativas, con el fin de fomentar la participación en padres de familia, estudiantes y docentes mediante la sensibilización, capacitación y orientación.

\section{Material y métodos}

\section{Diseño}

Para los fines de esta investigación, se realizó un estudio desde un enfoque empírico, se utilizó métodos descriptivos, analítico no experimental empleando instrumentos de recolección de datos como encuestas, Test de Apgar y la Ficha familiar, fichas que se centraron en la prevención y atención de conductas adictivas en adolescentes del cantón Jipijapa. Se seleccionó 4 ciudadelas de la periferia urbana del cantón, realizando un barrido casa a casa, identificando posibles adolescentes con consumo problemático. El análisis de la información se lleva a cabo con la elaboración de un informe donde se recogen las impresiones obtenidas en la sesión, incluyendo fragmentos de las trascripciones, cuyo contenido refuerza las opiniones emitidas.

\section{Resultados}

Tabla 1: Miembros de la familia por grupos de género y edades. 
La información de la población de estudio conformada por 427 personas, de las ciudadelas Luis Bustamante, 3 de mayo, Ricardo Loor y la FAE, es relevante ya que, según los resultados de las familias encuestadas, 245 hombres y 182 mujeres, esto nos indica que el género está jugando un rol preponderante al momento de
Vol. 5, Nro. 2, Publicado: 2021-12-31 evaluar el consumo de sustancias licitas e ilícitas, a su vez deja en evidencia que 140 niños y jóvenes, cursan los años de estudio de básica, mientras que 137 en bachillerato y $45 \mathrm{sin}$ escolaridad según indican, no cuentan con las oportunidades de ingreso a las instituciones de educación superior.

\begin{tabular}{|c|c|c|c|c|}
\hline VARIABLE & $\begin{array}{c}\text { Ciudadela Luis } \\
\text { Bustamante }\end{array}$ & $\begin{array}{c}\text { Ciudadela 3 } \\
\text { de Mayo }\end{array}$ & $\begin{array}{c}\text { Ciudadela, } \\
\text { Ricardo Loor }\end{array}$ & $\begin{array}{c}\text { Ciudadela la } \\
\text { FAE }\end{array}$ \\
\hline Hombre & 68 & 54 & 79 & 44 \\
\hline Mujer & 53 & 37 & 61 & 31 \\
\hline \multicolumn{5}{|c|}{ Escolaridad } \\
\hline Sin escolaridad & 18 & 8 & 13 & 6 \\
\hline Básica & 28 & 33 & 47 & 27 \\
\hline Bachillerato & 43 & 25 & 42 & 13 \\
\hline Superior & 14 & 10 & 24 & 7 \\
\hline Especialidad & 8 & 15 & 14 & 85 \\
\hline Total & 111 & 91 & 140 & \\
\hline
\end{tabular}

Tabla 2: Salud bucal y embarazo en adolescentes

Es alarmante lo que revela los resultados de esta investigación, el $80.4 \%$ de la población de estudio no tienen acceso a la atención odontológica, a nivel de Ecuador, considerando que la enfermedad periodontal sigue siendo el principal problema bucal que se relaciona con el embarazo.

\begin{tabular}{|c|c|c|c|c|}
\hline VARIABLE & $\begin{array}{c}\text { Ciudadela Luis } \\
\text { Bustamante }\end{array}$ & $\begin{array}{c}\text { Ciudadela 3 de } \\
\text { Mayo }\end{array}$ & $\begin{array}{c}\text { Ciudadela Ricardo } \\
\text { Loor }\end{array}$ & $\begin{array}{c}\text { Ciudadela La } \\
\text { FAE }\end{array}$ \\
\hline $\mathrm{Si}$ & 45 & 99 & 117 & 34 \\
\hline $\mathrm{No}$ & 35 & 2 & 14 & 49 \\
\hline Total & 80 & 101 & 131 & 83 \\
\hline \multicolumn{5}{|c|}{ Embarazos } \\
\hline $\mathrm{Si}$ & 0 & 2 & 4 & 24 \\
\hline $\mathrm{No}$ & 32 & 28 & 37 & 0 \\
\hline
\end{tabular}

Tabla 3: Factores de riesgos en la salud de la población de estudio

El riesgo implica la probabilidad de que la presencia de una o más características o factores incremente la aparición de consecuencias adversas para la salud, el proyecto de vida, la supervivencia personal o de otros, da una medida la necesidad de atención y la integralidad de los factores que se toman en cuenta, estos riesgos que demuestran las tablas 3 y 4, dejan claro que supone un riesgo de consumir sustancia ilícitas, ya que en las 4 ciudadelas encuestadas, todas coinciden con la alta tasa de desempleo, el analfabetismo, la desnutrición, malas condiciones de vivienda y más evidente el hacinamiento en el que viven. 


\begin{tabular}{|c|c|c|c|c|c|c|c|c|c|c|}
\hline \multicolumn{11}{|c|}{ Ciudadela Bustamante } \\
\hline \multicolumn{2}{|r|}{ Variables } & \multirow{2}{*}{$\begin{array}{c}\text { Pobreza } \\
\\
9\end{array}$} & \multirow{2}{*}{$\begin{array}{c}\text { Desempleo } \\
\text { o empleo } \\
\text { informal } \\
\text { del jefe de } \\
\text { familia } \\
13\end{array}$} & \multirow{2}{*}{$\begin{array}{c}\text { Analfabetismo } \\
\text { del padre o la } \\
\text { madre }\end{array}$} & \multirow{2}{*}{$\begin{array}{c}\begin{array}{c}\text { Desestructuración } \\
\text { familiar }\end{array} \\
\\
29\end{array}$} & \multirow{2}{*}{$\begin{array}{c}\begin{array}{c}\text { Violencia / } \\
\text { alcoholismo } \\
/ \\
\text { drogadicción }\end{array} \\
31\end{array}$} & \multirow{2}{*}{$\begin{array}{c}\begin{array}{c}\text { Malas } \\
\text { condiciones } \\
\text { de la } \\
\text { vivienda }\end{array} \\
23 \\
\end{array}$} & \multirow{2}{*}{$\begin{array}{c}\text { Hacinamiento } \\
\\
29\end{array}$} & \multirow{2}{*}{$\begin{array}{c}\text { Total } \\
\\
156 \\
\end{array}$} & \multirow{2}{*}{$\begin{array}{l}\% \\
70\end{array}$} \\
\hline 0 & Sin riesgo & & & & & & & & & \\
\hline 1 & $\begin{array}{l}\text { Riesgo } \\
\text { muy bajo }\end{array}$ & 8 & 8 & 6 & 0 & 1 & 6 & 2 & 31 & 14 \\
\hline 2 & $\begin{array}{l}\text { Riesgo } \\
\text { bajo }\end{array}$ & 10 & 7 & 2 & 0 & 0 & 3 & 1 & 23 & 10 \\
\hline 3 & $\begin{array}{l}\text { Riesgo } \\
\text { moderado }\end{array}$ & 5 & 4 & 2 & 0 & 0 & 0 & 0 & 11 & 5 \\
\hline 4 & $\begin{array}{l}\text { Riesgo } \\
\text { alto }\end{array}$ & 0 & 0 & 0 & 3 & 0 & 0 & 0 & 3 & 1 \\
\hline & Total & 32 & 32 & 32 & 32 & 32 & 32 & 32 & 224 & 100 \\
\hline \multicolumn{11}{|c|}{ Ciudadela 3 de Mayo } \\
\hline 0 & Sin riesgo & 12 & 13 & 22 & 28 & 30 & 22 & 28 & 155 & 74 \\
\hline 1 & $\begin{array}{l}\text { Riesgo } \\
\text { muy bajo }\end{array}$ & 4 & 5 & 0 & 0 & 0 & 1 & 2 & 12 & 6 \\
\hline 2 & $\begin{array}{l}\text { Riesgo } \\
\text { bajo }\end{array}$ & 11 & 6 & 4 & 0 & 0 & 7 & 0 & 28 & 13 \\
\hline 3 & $\begin{array}{l}\text { Riesgo } \\
\text { moderado }\end{array}$ & 2 & 5 & 4 & 0 & 0 & 0 & 0 & 11 & 5 \\
\hline 4 & $\begin{array}{l}\text { Riesgo } \\
\text { alto }\end{array}$ & 1 & 1 & 0 & 2 & 0 & 0 & 0 & 4 & 2 \\
\hline & Total & 30 & 30 & 30 & 30 & 30 & 30 & 30 & 210 & 100 \\
\hline \multicolumn{11}{|c|}{ Ricardo Loor } \\
\hline 0 & Sin riesgo & 19 & 21 & 30 & 38 & 39 & 27 & 37 & 211 & 74 \\
\hline 1 & $\begin{array}{l}\text { Riesgo } \\
\text { muy bajo }\end{array}$ & 5 & 7 & 1 & 0 & 2 & 7 & 4 & 26 & 9 \\
\hline 2 & $\begin{array}{l}\text { Riesgo } \\
\text { bajo }\end{array}$ & 12 & 7 & 6 & 0 & 0 & 7 & 0 & 32 & 11 \\
\hline 3 & $\begin{array}{l}\text { Riesgo } \\
\text { moderado }\end{array}$ & 4 & 4 & 2 & 0 & 0 & 0 & 0 & 10 & 3 \\
\hline 4 & $\begin{array}{l}\text { Riesgo } \\
\text { alto }\end{array}$ & 1 & 2 & 2 & 3 & 0 & 0 & 0 & 8 & 3 \\
\hline & Total & 41 & 41 & 41 & 41 & 41 & 41 & 41 & 287 & 100 \\
\hline \multicolumn{11}{|c|}{ La FAE } \\
\hline 0 & Sin riesgo & 8 & 13 & 18 & 20 & 22 & 15 & 20 & 116 & 69 \\
\hline 1 & $\begin{array}{l}\text { Riesgo } \\
\text { muy bajo }\end{array}$ & 6 & 5 & 0 & 1 & 2 & 5 & 3 & 22 & 13 \\
\hline 2 & $\begin{array}{l}\text { Riesgo } \\
\text { bajo }\end{array}$ & 7 & 5 & 3 & 0 & 0 & 4 & 1 & 20 & 12 \\
\hline 3 & $\begin{array}{l}\text { Riesgo } \\
\text { moderado }\end{array}$ & 2 & 1 & 3 & 0 & 0 & 0 & 0 & 6 & 4 \\
\hline 4 & $\begin{array}{l}\text { Riesgo } \\
\text { alto }\end{array}$ & 1 & 0 & 0 & 3 & 0 & 0 & 0 & 4 & 2 \\
\hline & Total & 24 & 24 & 24 & 24 & 24 & 24 & 24 & 168 & 100 \\
\hline
\end{tabular}


Tabla 4: Riesgos Biológicos

\begin{tabular}{|c|c|c|c|c|c|c|c|c|}
\hline \multicolumn{9}{|c|}{ Luis Bustamante } \\
\hline & Variables & $\begin{array}{l}\text { Personas } \\
\text { con } \\
\text { vacunació } \\
\text { n } \\
\text { incomplet } \\
\text { a. }\end{array}$ & $\begin{array}{l}\text { Personas con } \\
\text { malnutrición } \\
\text { (sobrepeso o } \\
\text { desnutrición) }\end{array}$ & $\begin{array}{l}\text { Personas } \\
\text { con } \\
\text { enfermeda } \\
\text { d de } \\
\text { impacto }\end{array}$ & $\begin{array}{l}\text { Embarazad } \\
\text { as con } \\
\text { problemas }\end{array}$ & $\begin{array}{l}\text { Personas } \\
\text { con } \\
\text { discapacid } \\
\text { ad }\end{array}$ & $\begin{array}{l}\text { Personas } \\
\text { con } \\
\text { problemas } \\
\text { mentales }\end{array}$ & $\begin{array}{c}\text { Tota } \\
\mathbf{l}\end{array}$ \\
\hline $\mathbf{0}$ & Sin riesgo & 27 & 21 & 21 & 28 & 28 & 30 & 155 \\
\hline 1 & $\begin{array}{l}\text { Riesgo muy } \\
\text { bajo }\end{array}$ & 0 & 0 & 6 & 0 & 2 & 0 & 8 \\
\hline 2 & Riesgo bajo & 2 & 9 & 3 & 2 & 0 & 0 & 16 \\
\hline 3 & $\begin{array}{l}\text { Riesgo } \\
\text { moderado }\end{array}$ & 4 & 3 & 2 & 0 & 1 & 0 & 10 \\
\hline 4 & Riesgo alto & 1 & 0 & 4 & 0 & 0 & 0 & 5 \\
\hline & Total & 34 & 33 & 36 & 30 & 31 & 30 & 194 \\
\hline \multicolumn{9}{|c|}{ Ciudadela 3 de Mayo } \\
\hline $\mathbf{0}$ & Sin riesgo & 27 & 21 & 21 & 28 & 28 & 30 & 155 \\
\hline 1 & $\begin{array}{l}\text { Riesgo muy } \\
\text { bajo }\end{array}$ & 0 & 0 & 6 & 0 & 2 & 0 & 8 \\
\hline 2 & Riesgo bajo & 2 & 9 & 3 & 2 & 0 & 0 & 16 \\
\hline 3 & $\begin{array}{l}\text { Riesgo } \\
\text { moderado }\end{array}$ & 3 & 0 & 2 & 0 & 0 & 0 & 5 \\
\hline 4 & Riesgo alto & 0 & 2 & 3 & 0 & 1 & 0 & 6 \\
\hline & Total & 33 & 32 & 35 & 30 & 31 & 30 & 190 \\
\hline \multicolumn{9}{|c|}{ Ricardo Loor } \\
\hline 0 & Sin riesgo & 34 & 31 & 28 & 41 & 40 & 41 & 211 \\
\hline 1 & $\begin{array}{l}\text { Riesgo muy } \\
\text { bajo }\end{array}$ & 4 & 8 & 4 & 2 & 1 & 0 & 19 \\
\hline 2 & Riesgo bajo & 1 & 2 & 8 & 0 & 0 & 0 & 11 \\
\hline 3 & $\begin{array}{l}\text { Riesgo } \\
\text { moderado }\end{array}$ & 2 & 0 & 2 & 2 & 0 & 0 & 4 \\
\hline 4 & Riesgo alto & 2 & 2 & 1 & 0 & 0 & 0 & 1 \\
\hline & Total & 41 & 41 & 41 & 41 & 41 & 41 & 246 \\
\hline \multicolumn{9}{|c|}{ La FAE } \\
\hline $\mathbf{0}$ & Sin riesgo & 23 & 17 & 15 & 24 & 21 & 23 & 123 \\
\hline 1 & $\begin{array}{l}\text { Riesgo muy } \\
\text { bajo }\end{array}$ & 0 & 2 & 1 & 0 & 1 & 0 & 4 \\
\hline 2 & Riesgo bajo & 1 & 4 & 7 & 0 & 2 & 1 & 15 \\
\hline 3 & $\begin{array}{l}\text { Riesgo } \\
\text { moderado }\end{array}$ & 0 & 1 & 1 & 0 & 0 & 0 & 2 \\
\hline 4 & Riesgo alto & 0 & 0 & 0 & 0 & 0 & 0 & 0 \\
\hline & Total & 24 & 24 & 24 & 24 & 24 & 24 & 144 \\
\hline
\end{tabular}

\section{DISCUSIÓN}

Los resultados del estudio apoyan la importancia crítica de los factores de riesgo familiares económicos y biológicos, familiares para el desarrollo de abuso de sustancias en la juventud, características que ponen de manifiesto a la adolescencia como un período crítico para los efectos nocivos de la exposición al consumo de sustancias 
Periodo. Julio - Diciembre 2021

ilícitas. El desarrollo de estrategias de prevención e intervención inmediata dirigidas a los adolescentes con alto riesgo es imperante en este grupo (Martinez Loredo, 2016)

Así en este estudio se observa como la familia es el escenario donde los adolescentes adquieren los recursos psicológicos con los que enfrentará esta etapa, estos recursos se traducen tanto en la capacidad para evaluar situaciones de la vida, esto es, anticipando y planeando la conducta a seguir para una mejor adaptación, como en la capacidad para reconocer y expresar sus reacciones afectivas. Si la estructura familiar no se desestabiliza o, si los padres no asumen su rol formativo de la manera más idónea, el individuo llegará a la adolescencia sin las herramientas necesarias para enfrentarse a la vida a través de la toma de decisiones correctas y convenientes.

La estructura familiar juega un papel importante en el desarrollo del niño. (Loke Yoen \& Mak Wah, 2013) En el caso de las familias monoparentales, adolescentes y jóvenes que provienen de familias destructuradas tienen mayor probabilidad de caer en las drogas, ya que existe la insatisfacción de roles paternos lo que conlleva a una comunicación disminuida.

Teniendo en cuenta lo anteriormente mencionado se llega a la conclusión que esta fase en la etapa adolescente, en la cual el estancamiento o la no superación de algunas instancias y de algunos estadios pueden llegar a generar el sentimiento de vulnerabilidad, y así mismo de indefensión, al haber dificultad en la relación y el vínculo con los padres la ruta de la adicción es algo permanente, hay que considerar que la primera adicción vigente en el cuerpo es la masturbación de una manera más personal o más privada, sin embargo las adicciones restantes, como a hábitos objétales y a toxicomanías
Publicado: Vol. 5, Nro. 2, 2021-12-31

y alcohol, son el escape o los encargados de conseguir la sujeción o sustitución del objeto,

Por último, se puede ver de una manera tangible que la persona adicta no es capaz de cuidar de sí mismo, pues aquel trauma por decir así, se inició por la indefensión por parte sus padres, y ellos buscan sin saber la sujeción o suplantación de estos en búsqueda del objeto idea.

\section{Conclusiones}

- El trabajo preventivo es viable desde el punto de vista educativo e integrador, asumiéndose como una dimensión de la educación de prevención del consumo de alcohol, lo cual permite desarrollar un modelo de compartimiento sano y saludable que contribuya de forma adecuada al crecimiento profesional de los estudiantes.

- La propuesta de las actividades que se pretenden desarrollar y su aplicación se constituirá en una vía eficaz para poder desarrollar actividades extracurriculares, tomando en cuenta la participación fundamental que realizan los docentes en la formación de los adolescentes y así propiciar un comportamiento que contribuya a un sano desarrollo y vida de los estudiantes.

- El confinamiento a raíz de la pandemia, ha jugado un papel muy importante puesto que, el mantenerse en confinio, limito a los adolescentes a salir de casa, a asistir a reuniones, convivir con amistades y consumir algun tipo de sustancias. Es importante recordar que, el consumo de sustancias en adolescentes en un problema de salud con gran efecto en el desarrollo biopsicosocial del adolescente. 
Referencias

Francoises Contrera, Esguerra, G., Espinosa, J. C., Gutiérrez, C., \& Fajardo, L. (2006). Calidad de vida y adhesión al tratamiento en pacientes con insuficiencia renal crónica en tratamiento de hemodiálisis. Universitas Psychologica. Recuperado el 04 de 06 de 2021, de http://pepsic.bvsalud.org/scielo.php?script=sci_arttext\&pid=S1657-92672006000300005

Gómez Cobos, E. (2018). Adolescentes y familia. Revista Intercontinental de Psicología y Educacion, 12. Recuperado el 10 de 09 de 2021, de https://www.redalyc.org/pdf/802/80212387006.pdf

Guaranda Moreira , J. E. (2018). Consumo problemático de sustancias adictivas en adolescentes. Universidad Estatal del Sur de Manabí, ipijapa. Recuperado el 10 de 09 de 2021, de http://repositorio.unesum.edu.ec/bitstream/53000/1354/1/UNESUM-ECUADOR-ENFERMERIA-201826.pdf

Sanz Turrado, M., Garrido Pérez , L., \& Caro Dominguez, C. (2017). Factores que influyen en la satisfacción del paciente de diálisis con enfermería. Scielo, 20(1). Recuperado el 08 de 06 de 2021, de https://scielo.isciii.es/scielo.php?script=sci_arttext\&pid=S2254-28842017000100009

Bermeo Minchala, M. L., \& Jimenez Yora, J. V. (2014). Evaluacion de la calidad de vida de los pacientes con Insuficicencia Renal Cronica sometidos a hemodialisis. Azoguez. Recuperado el 04 de 06 de 2021, de http://dspace.ucuenca.edu.ec/bitstream/123456789/21563/1/Tesis\%20Pregrado.pdf

Cid Monckton, P., \& Pedrão , L. J. (2011). Protective and family risk factors related to adolescent drug use. Revista Latino-Americana de Enfermagem, 15. Recuperado el 10 de 09 de 2021, de https://doi.org/10.1590/S0104-11692011000700011

Consejo de Organizaciones Internacionales de las Ciencias Medicas. (2016). Pautas éticas internacionales para la investigacion relacionada con la salud con seres vivos. Organizacion Mundial de la Salud, Ginebra. Recuperado el 3 de 06 de 2021, de https://iris.paho.org/bitstream/handle/10665.2/34457/9789290360902-spa.pdf?sequence=5\&isAllowed=y

David, R. G., \& Sanjuán Hernández, D. (2018). Satisfacción percibida por los pacientes en el servicio de hemodiálisis del hospital general de La Palma. Redyalic, 15. Recuperado el 2021 de 07 de 28, de https://www.redalyc.org/pdf/3598/359833150002.pdf

Jimenez, T. I., Murgui, S., Estevez, E., \& Musitu, G. (2007). Comunicacion familiar y comportamientos delictivos en adolescentes de Madrid, el doble rol de mediador. Redialyc, 12. Recuperado el 10 de 09 de 2021, de https://www.redalyc.org/pdf/805/80539303.pdf

Observatorio de Drogas de Colombia. (09 de 2018). Observatorio de Drogas Colombia. Recuperado el 10 de 09 de 2021, de http://www.odc.gov.co/PUBLICACIONES/ArtMID/4214/ArticleID/5929/Informe-MundialSobre-las-Drogas-2014

OPS. (10 de 03 de 2015). Organizacion Panamericana de la Salud. Recuperado el 04 de 08 de 2021, de https://www3.paho.org/hq/index.php?option=com_content\&view=article\&id=10542:2015-opsomssociedad-latinoamericana-nefrologia-enfermedad-renal-mejorar-tratamiento\&Itemid=1926\&lang=es

Ponce Bacusoy, M. T., Suarez Martinez, F. J., \& Pincay Parrales, E. G. (2021). Trastornos del comportamiento asociados a sustancias psicoactivas en estudiantes de bachillerato de la Unidad Educativa Alejo Lazcano. Revista Ciencias, 16. doi:http://dx.doi.org/10.23857/dc.v7i1.1867

Sánchez Hernández, C., Rivadeneyra Espinoza, L., \& Aristil Chery, P. M. (2016). Calidad de vida en pacientes bajo hemodiálisis en un hospital público de Puebla, México. Scielo, 20(3), 15. Recuperado el 10 de 08 de 2021, de http://scielo.sld.cu/scielo.php?pid=S1025-02552016000300006\&script=sci_arttext\&tlng=en

Tumbaco Quimis, G. A. (2018). Consumo problemático de sustancias adictivas en los adolescentes. Jipijapa. Recuperado el 0910 de 2021, de http://repositorio.unesum.edu.ec/bitstream/53000/1347/1/UNESUMECUADOR-ENFERMERIA-2018-19.pdf 